\title{
Omega 3 fatty acid supplementation after myocardial infarction: a systematic review and meta-analysis
}

Federico Popoff ${ }^{1 * \dagger}$, Giselle Balaciano ${ }^{2^{*}+}$ D, Ariel Bardach ${ }^{3}$, Daniel Comandé ${ }^{3}$, Vilma Irazola ${ }^{3}$, Hugo Norberto Catalano ${ }^{1}$ and Ariel Izcovich ${ }^{1}$

\begin{abstract}
Background: The purpose of this review is to examine the effect of Omega-3 Fatty acids on mortality, morbidity, and adverse events in patients with acute myocardial infarction (AMI).

Methods: Data Sources: MEDLINE, EMBASE, and the Cochrane Library through May 2018. Study Selection: Randomized Controlled trials (RCT). Certainty of evidence was assessed with the GRADE system. Interventions: omega 3 fatty acids against placebo or no treatment. Primary and secondary outcomes: All-cause death, cardiovascular death, new AMI, stroke, need for therapeutic angioplasty or By-pass, new diagnosis of cancer and incidence of adverse events.

Results: For the efficacy endpoints we included 10 RCT (24,414 patients). Omega 3 fatty acids probably make little or no difference to all-cause mortality (4 studies 9141 patients RR 1.06 - Cl95\% 0.90 to 1.27, moderate certainty), cardiovascular mortality (3 studies 4304 patients RR 0.93 - Cl95\% 0.63 to 1.37, moderate certainty), new AMI (RR 1.24 Cl95\% 0.71 to 2.14 - moderate certainty), any cardiovascular event (RR $0.9595 \% \mathrm{Cl} 0.86$ to 1.05 ; low certainty due to risk of bias and imprecision), and stroke (RR $1.295 \% \mathrm{CCl}$ 0,66-2,19 - moderate certainty). Regarding adverse events, we are uncertain if Omega 3 fatty acids improve/reduce non severe adverse events (RR 1.39 95\% Cl 0.36 to 5.34; very low certainty). There is probably little or no difference in the outcome suspension due to adverse events (RR 1. 19 Cl 95\% 0.97 to 1.47; moderate certainty).
\end{abstract}

Conclusions: For adult patients with AMl, omega 3 fatty-acids probably yield no benefit to patient important outcomes. Keywords: Omega 3 fatty acid, Polyunsaturated fatty acids, Myocardial infarction, Secondary prevention, Systematic review

\section{Background}

n-3PUFAs are a family of polyunsaturated fatty acids, named as such because of the positioning of the first double carbon bond on the third atom from the methyl end of the acyl chain. Dietary sources of Omega 3 include certain nuts and seeds, such as walnuts, flaxseed and rapeseed (canola) oil, fatty fish, some white fish, shellfish and other seafood such as seaweed, and certain eggs and animal products, depending on the animal's diet.

\footnotetext{
* Correspondence: federicopopoff@gmail.com; giselle.balaciano@gmail.com ${ }^{\dagger}$ Federico Popoff and Giselle Balaciano contributed equally to this work. ${ }^{1}$ Ministry of health, Buenos Aires, Argentina

${ }^{2}$ National Ministry of Health of Argentina, Hospital Alemán, Buenos Aires, Argentina

Full list of author information is available at the end of the article
}

Proposed benefits of omega 3 fatty acids include lowering of blood pressure, reducing serum triglyceride concentration, increasing plaque stability and improving endothelial function [1-4]. In the context of previous acute myocardial infarction the mentioned omega 3 fatty acids plaque stabilization properties could result in significant benefits [4-7].

Multiple randomized controlled trials (RCT) evaluating the efficacy and safety safety of fatty acids have been published and their results summarized in different systematic reviews. However those published analysis have important limitations as we describe in the Additional file 1: Appendix 1. Furthermore, none of them performed a differential AMI patients analysis. In this context, we consider that a new systematic review (SR) is justified. The present systematic

(c) The Author(s). 2019 Open Access This article is distributed under the terms of the Creative Commons Attribution 4.0 International License (http://creativecommons.org/licenses/by/4.0/), which permits unrestricted use, distribution, and 
review and meta-analysis aimed to improve estimations and to assess whether dietary or supplemental omega 3 fatty acids affects total or cardiovascular mortality in the context of myocardial infarction secondary prevention.

\section{Methods}

This research is not a clinical trial and therefore does not need to be registered.

\section{Search strategy and elegibility criteria}

We searched for randomized controlled trials comparing omega 3 fatty acids against placebo or no treatment in the following literature databases, regardless of publication status and without language restrictions: the Cochrane Central Register of Controlled Trials from the Cochrane Library, MEDLINE, EMBASE, Epistemonikos and LILACS from inception until May 2018.Details of the full search strategies are provided in the Additional file 1: Appendix 2. Our gray-literature search included searches in Grey Matters Tool [8]. We also searched the Canadian Agency for Drugs and Technology in Health, Google Scholar, Trip Database, National Institute for Health and Care Excellence, McMaster University, McMaster Health Forum, PROSPERO, ClinicalTrials.gov, and manually examined the reference lists of all reviews identified.

As for the inclusion criteria, we included RCTs of adults that suffered a myocardial infarction (according to the study's definition) and were randomized to receive omega 3-fatty acid supplementation at doses greater than or equal to $400 \mathrm{mg}$ daily versus placebo/No treatment. The treatment should have started within 6 weeks after the initial diagnosis of the myocardial infarction. We considered any mode of administration of the intervention, such as dietary supplementation (fish oils, soya bean oils, seeds, refined EPA, DHA, ALA) or, oil or capsule form or as foodstuffs. To be eligible, studies had to report at least one of the following outcomes: All-cause death, cardiovascular death, new acute myocardial infarction, stroke, need for therapeutic angioplasty or By-pass, new diagnosis of cancer and incidence of adverse events.

\section{Study selection and data extraction}

Two investigators (G.B. and F.P.) independently reviewed the titles and abstracts identified and full texts of included articles in order to determine eligibility using the EROS tool for systematic reviews early phases [9]. Disagreements or uncertainties were resolved by consensus of the whole team with an additional investigator (A.I.). We accepted the primary authors' definition of AMI, stroke, adverse event and serious adverse event.

The risk of bias was assessed independently by two reviewers on an outcome by outcome basis using a modification of the Cochrane Risk of Bias Tool which considers, sequence generation, allocation concealment, blinding, number of patients with missing outcome data, selective outcome reporting, and other sources of bias [10]. We used the Grading of Recommendations Assessment, Development and Evaluation system to assess the certainty of the effect (also known as quality of evidence or confidence in evidence) for each outcome and for the entire body of evidence $[11,12]$. Certainty of the effect takes into consideration the study design (in this case, randomized clinical trials); risk of bias, precision, consistency, directness of the evidence; and the probability of publication bias [13].

\section{Statistical analysis}

We analyzed the data using Review Manager, version 5.3 (Cochrane Collaboration). We used random-effects models for all analyses (Mantel-Haenszel risk ratios [RRs] for dichotomous outcomes) since significant heterogeneity was expected. Publication bias was assessed through visual inspection of funnel plots (Additional file 1: Appendix 3) and the subjective impression of the reviewers (G.B., F.P. and A.I.) considering the size of the included studies and sponsorship. We also contacted investigators to consult whether they had knowledge of other potentially relevant unpublished trials.

We used Cochrane's test for heterogeneity to determine whether studies in a meta-analysis evaluated the same underlying sizes of effect. We used the $\mathrm{I}^{2}$ statistic to test the degree of heterogeneity among studies (the proportion of total observed variability due to genuine variation rather than random error within studies) [14].

We planned a priori the following subgroup analyses as possible explanations for heterogeneity: 1) type of fatty acid: eicosapentaenoic acid and docosahexaenoic acid versus alpha-linolenic acids with a postulated larger effect for the latter $[9,15]$; 2) Dose effect: high dose (consumption of more than $4.5 \mathrm{~g}$ daily) of Omega 3 fatty acids could be associated with a larger treatment benefit; 3) Type of omega 3: synthetic omega 3 (in comparison with dietary recommendations with increased omega 3 fatty acid intake) could be associated with a larger treatment benefit; 4) Risk of bias: Studies with high risk of bias could be associated with a larger treatment benefit. We visually analyzed the results of each subgroup comparison and additionally tested for interaction by using a chi-square significance test [14].

\section{Dealing with missing data}

For the primary analysis we used a complete case-analysis approach, i.e. we excluded participants considered to have missing data. For those outcomes in which a clinically significant effect was observed (Relative risk C195\% not including 1 ), we performed a sensitivity analysis to challenge the possibility of risk of bias due to missing data following the approach described by Guyatt et.al $[16,17]$ (complete 
description of the implemented sensitivity analysis is available in Additional file 1: Appendix 4).

\section{Patient and public involvement}

Patients were not involved in this review.

\section{Results}

\section{Study characteristics}

We identified 610 potentially relevant records. After screening titles, abstracts and full texts we included 11 publications for quantitative analysis (Fig. 1.).

The characteristics of the 11 RCTs included are summarized in Table 1. In six [18-23] of them the intervention was implemented in the form of dietary recommendations while EDH-EPA (synthetic form of omega 3 fatty acids) was implemented in the remaining four [24-28].

\section{Risk-of-Bias assessment}

In five of the included studies [18, 22, 25-28], patients, investigators and outcome assessors were blinded. Four of those studies were judged as low risk of bias as no additional methodological issues were noted. Regarding the remaining studies, six were judged as to carry moderate or high risk of bias (Fig. 2). Although one of the included studies had no apparent methodological limitations, we decided to judge it as high risk of bias [27] because the author was accused of misconduct and data fabrication in two different trials in which he was involved [30, 31]. We assumed the trials to have important lost to follow-up when the authors did not offer enough information to analyze the impact of missing data or if the performed sensitivity analysis significantly altered the effect estimate or the confidence interval for each outcome. (Additional file 1: Appendix 4).

\section{All-cause mortality}

All the included trials addressed this outcome. Omega 3 fatty acids reduced the risk of all-cause mortality (RR 0.86, CI 95\% 0.72 to 1.02). Considering the basal risk (risk without the intervention) as the mean of the risk in the control groups of the included RCT, the mortality reduction was $1.4 \%$ (C195\% 2.5 to 0 ) at a mean follow up of 3 years. We judged the certainty in the estimates of

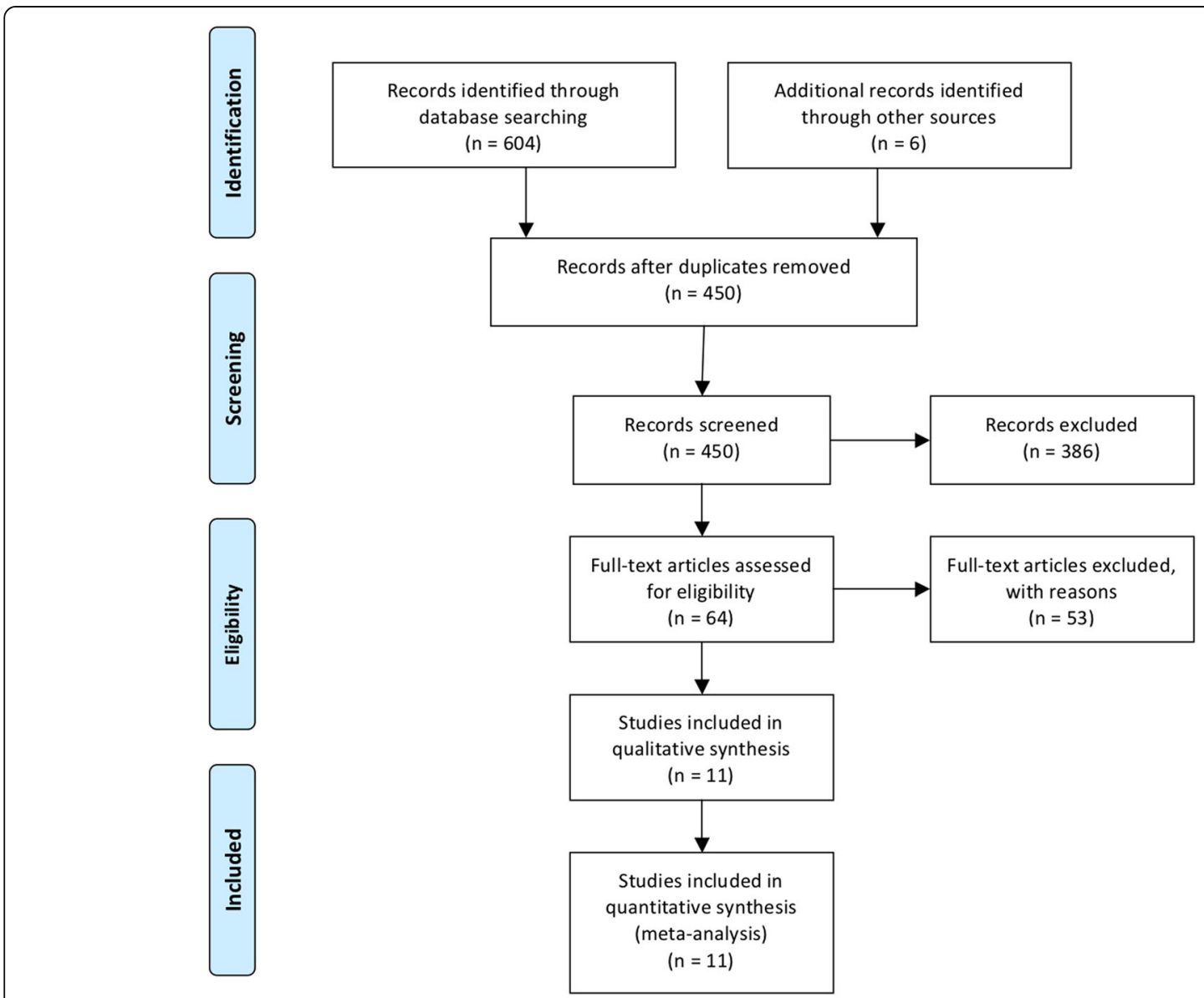

Fig. 1 Flow chart of study selection 


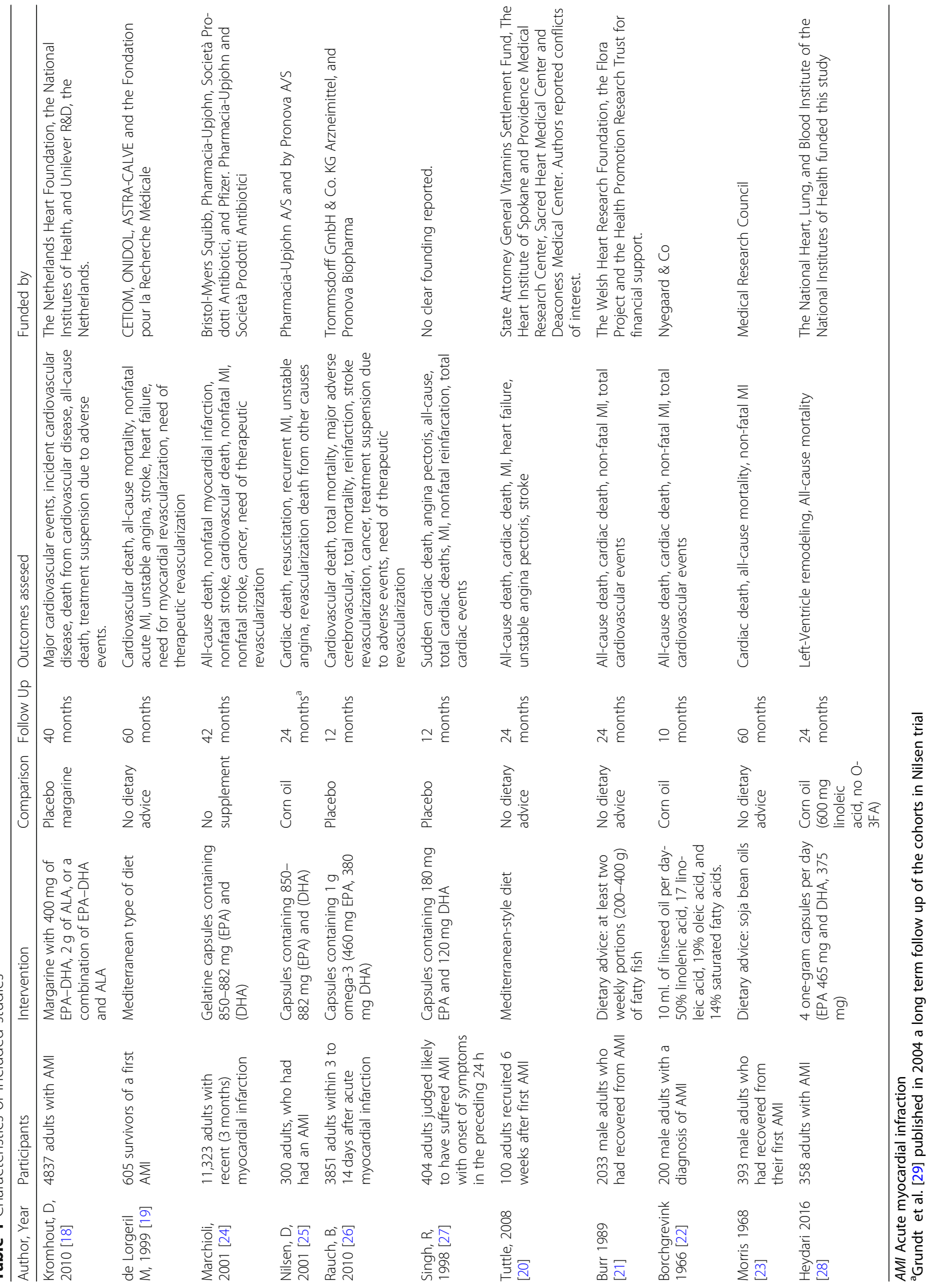




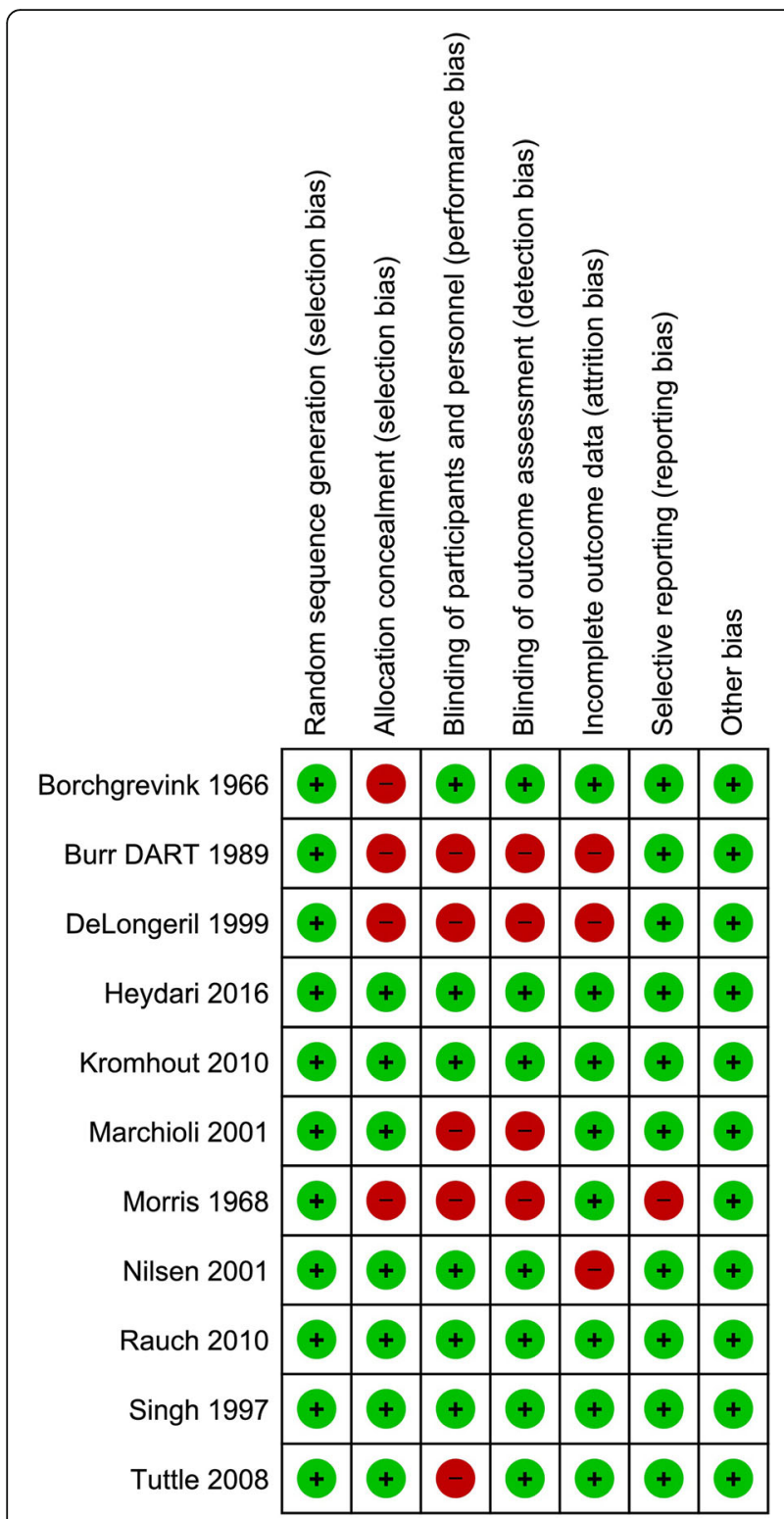

Fig. 2 Risk-of-Bias of included studies

effect as low due to risk of bias, imprecision and inconsistency ( $\mathrm{I}^{2} 85 \%$ ) (Fig. 3), (Table 2).

\section{Cardiovascular mortality}

Nine of the included trials addressed this outcome. Omega 3 fatty acids reduced the risk of cardiovascular mortality (RR 0.77, CI 95\% 0.65 to 0.91). Considering the basal risk as the mean of the risk in the control groups of the included RCT, the cardiovascular mortality reduction was $1.5 \%$ (CI95\% 2.3 to 0.6$)$ at a mean follow up of approximately 3 years. We judged the certainty in the estimates of effect as low due to risk of bias and inconsistency (Fig. 4), (Table 2).

\section{Acute myocardial infarction}

Seven of the included trials addressed this outcome. Omega 3 fatty acids reduced the risk of Myocardial infarction (RR 0.77, CI 95\% 0.6 to 0.99). Considering the basal risk as the mean of the risk in the control groups of the included RCT, the cardiovascular mortality reduction was $2.2 \%$ (CI95\% 3.8 to 0.1 ) at a mean follow up of approximately 3 years. We judged the certainty in the estimates of effect as low due to risk of bias, imprecision and inconsistency) (Fig. 5), (Table 2).

\section{Stroke}

Five of the included trials addressed this outcome. Omega 3 fatty acids did not reduce the risk of stroke (RR 1.2, CI 95\% 0.66 to 2.19). Considering the basal risk as the mean of the risk in the control groups of the included RCT a marginal increase in the risk of stroke was observed RD $0.2 \%$ (CI95\% - 0.4 to $1.4 \%$ ) at a mean follow up of approximately 3 years. We judged the certainty in the estimates of effect as moderate due to imprecision (Fig. 6), (Table 2).

\section{Need to therapeutic revascularization}

Three of the included trials addressed this outcome. Omega 3 fatty acids did not reduce the risk of therapeutic revascularization (RR 1.0 CI 95\% 0.91). Considering the basal risk as the mean of the risk in the control groups of the included RCT no differences in the need of therapeutic revascularization were observed RD $0 \%$ (CI95\% -1.9 to $2.4 \%$ ) at a mean follow up of approximately 3 years. We judged the certainty in the estimates of effect as moderate due to imprecision (Fig. 7), (Table 2).

\section{Treatment suspension due to adverse events}

Two of the included trials addressed this outcome. Omega 3 fatty acids increased the relative risk of treatment suspension due to adverse effects (RR 1.19 CI 95\% 0.97 to 1.47). Considering the basal risk as the mean of the risk in the control groups of the included RCT no differences in the risk of treatment suspension due to adverse effects was observed RD 0.3\% (CI95\% - 0.1 to $0.7 \%$ ) at a mean follow up of approximately 3 years. We judged the certainty in the estimates of effect as moderate due to imprecision (Fig. 8), (Table 2).

\section{Cancer}

Two of the included trials addressed this outcome. Omega 3 fatty acids increased the relative risk of cancer (RR 1.25 CI 95\% 0.94 to 1.66). Considering the basal risk as the mean of the risk in the control groups of the included RCT only a marginal increase in cancer occurrence was observed RD $0.3 \%$ (CI95\% -0.1 to $0.7 \%$ ) at a mean follow up of approximately 3 years. We judged the certainty in the estimates of effect as very low due to imprecision 


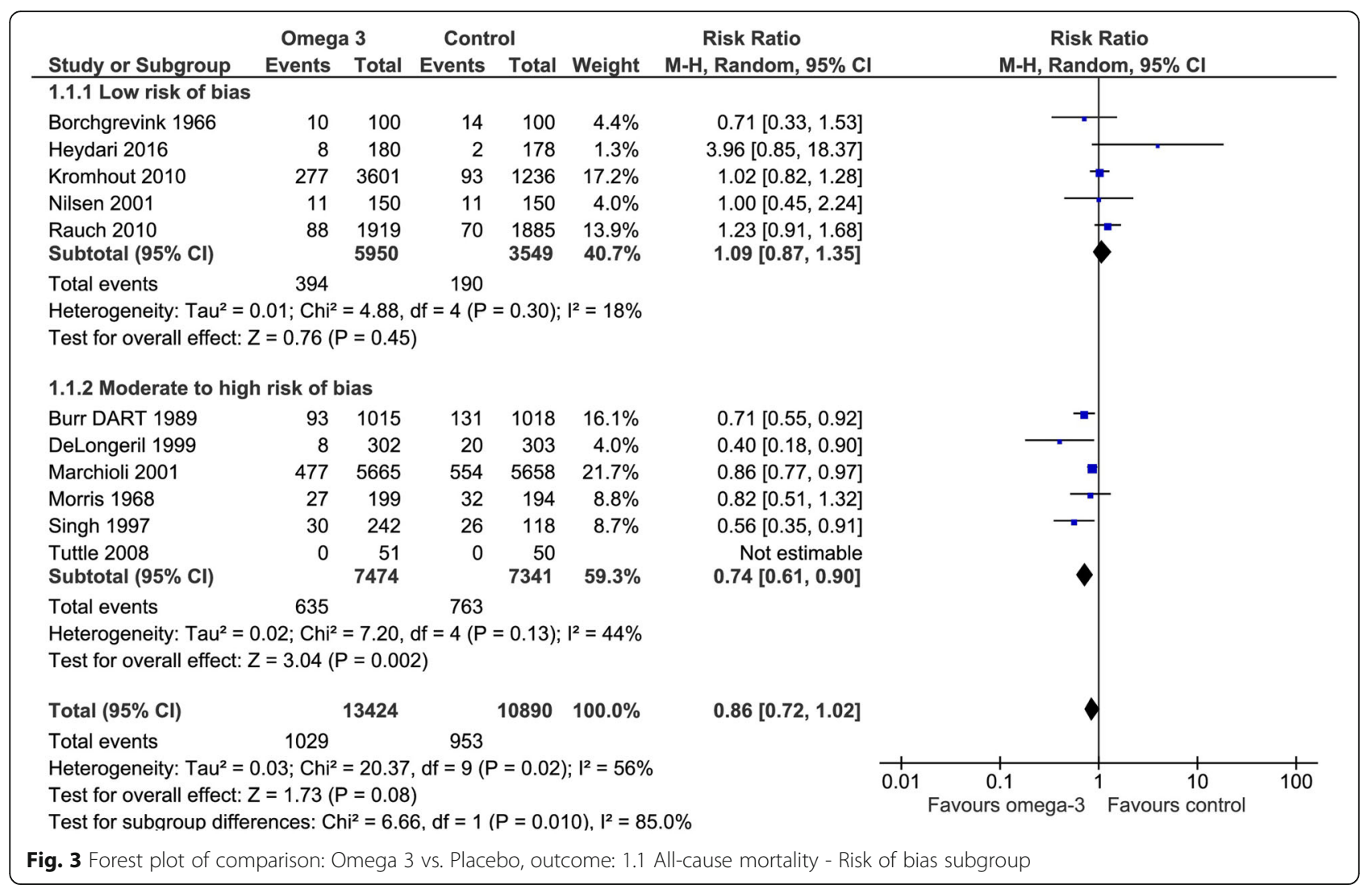

and indirectness (Fig. 9). The results are shown in a Summary of Finding Table (Table 2).

\section{Subgroup analysis}

Inconsistency was observed for overall mortality, cardiovascular mortality and myocardial infarction outcomes. In performing the prespecified risk of bias subgroup analysis we observed that the benefits (mortality, cardiovascular mortality and MI reduction), suggested by the overall pooled results, were not present when pooling the subgroup of studies in which the intervention was applied in a blinded fashion (low risk of bias). Although the test for subgroup differences was only statistically significant for overall mortality $(p<0.05)$ visual inspection of the forest plots suggest a similar subgroup effect for the three outcomes. The Fatty acid type subgroup analysis also showed a possible differential effect (less cardiovascular mortality) when EPA-DHA was implemented in comparison to ALA (RR 0.66, IC95\% 0.351.27 for ALA vs. RR 0.82, IC95\% 0.72-094) for EPA + DHA. No significant differences were observed when different doses were used.

We then decided to include, in the summary of findings tables, both the results of the overall pooled estimates and the results of the blinded trials pooled estimates (Table 2). We used primarily a $4 \mathrm{mg}$ threshold to evaluate the effect of the dose of omega 3 fatty acid which showed no effect on the evaluated outcomes. A secondary analysis using a $1 \mathrm{mg}$ threshold showed also no impact on those outcomes.

\section{Discussion}

This systematic review provides moderate quality of evidence that Omega 3 fatty acids do not significantly reduce mortality or major vascular events in patients with acute myocardial infarction. Although the overall estimates of effects analysis suggested a reduction in mortality and AMI recurrence, based on the results of the subgroup analysis we consider that this finding is probably biased. Hence our main conclusions are based in the pooled estimates provided by the blinded RCT (low risk of bias subgroup). Nevertheless, we decided to report both estimates (overall and low risk of bias subgroup) in order to supply decision makers with all the information (see Table 2). Regarding adverse events, particular concern has been raised by the possibility that the intervention could cause cancer. This hypothesis was drawn based on the observation that omega- 3 fats capsules could contain high levels of various toxic compounds such as mercury, polychlorinated biphenyl and dioxins [32-37]. Our results provide very low quality of evidence suggesting that omega 3 may increase the risk of cancer but the scarce number of events and the limited time of follow up make it difficult to draw definite 


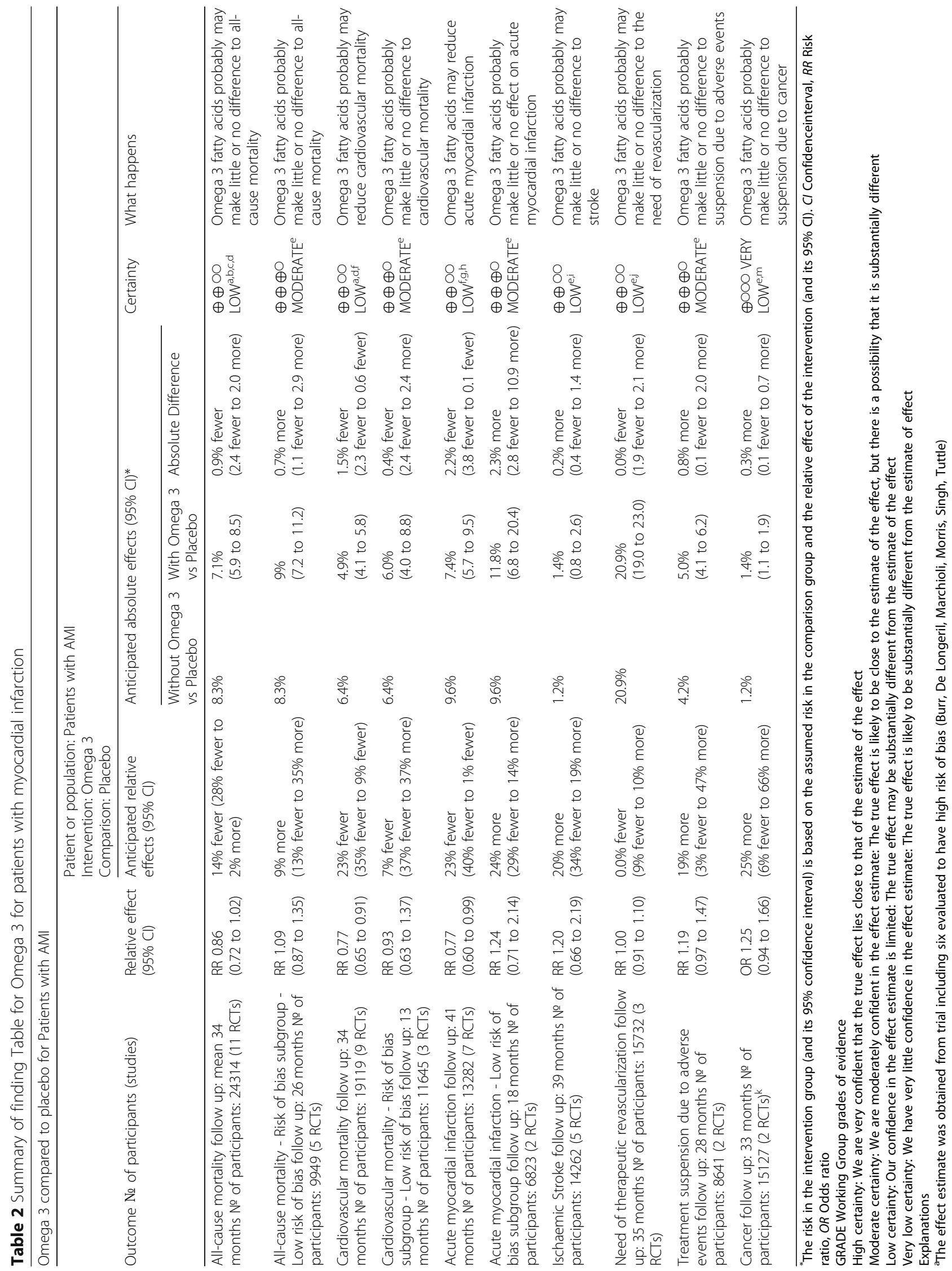




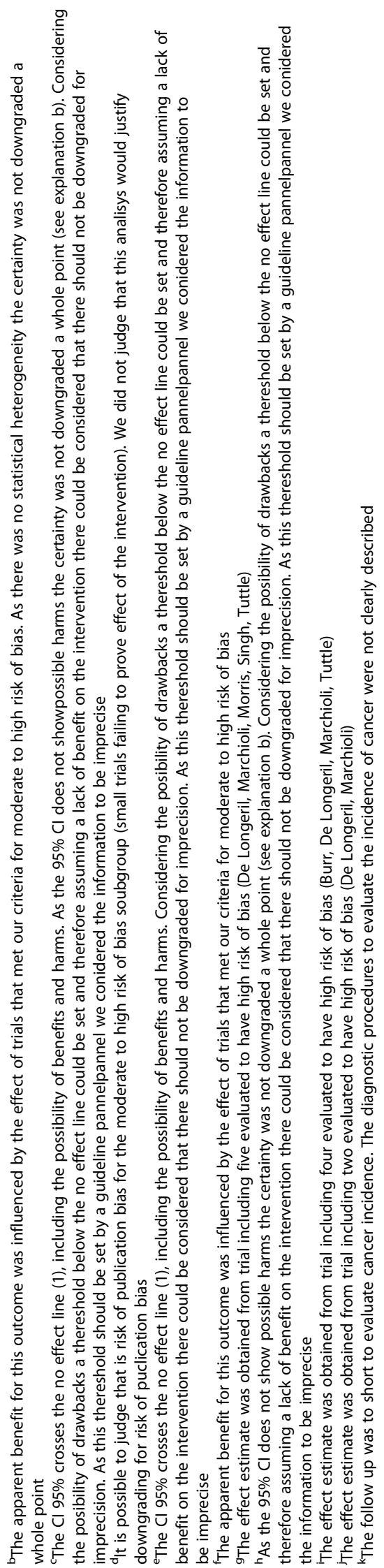




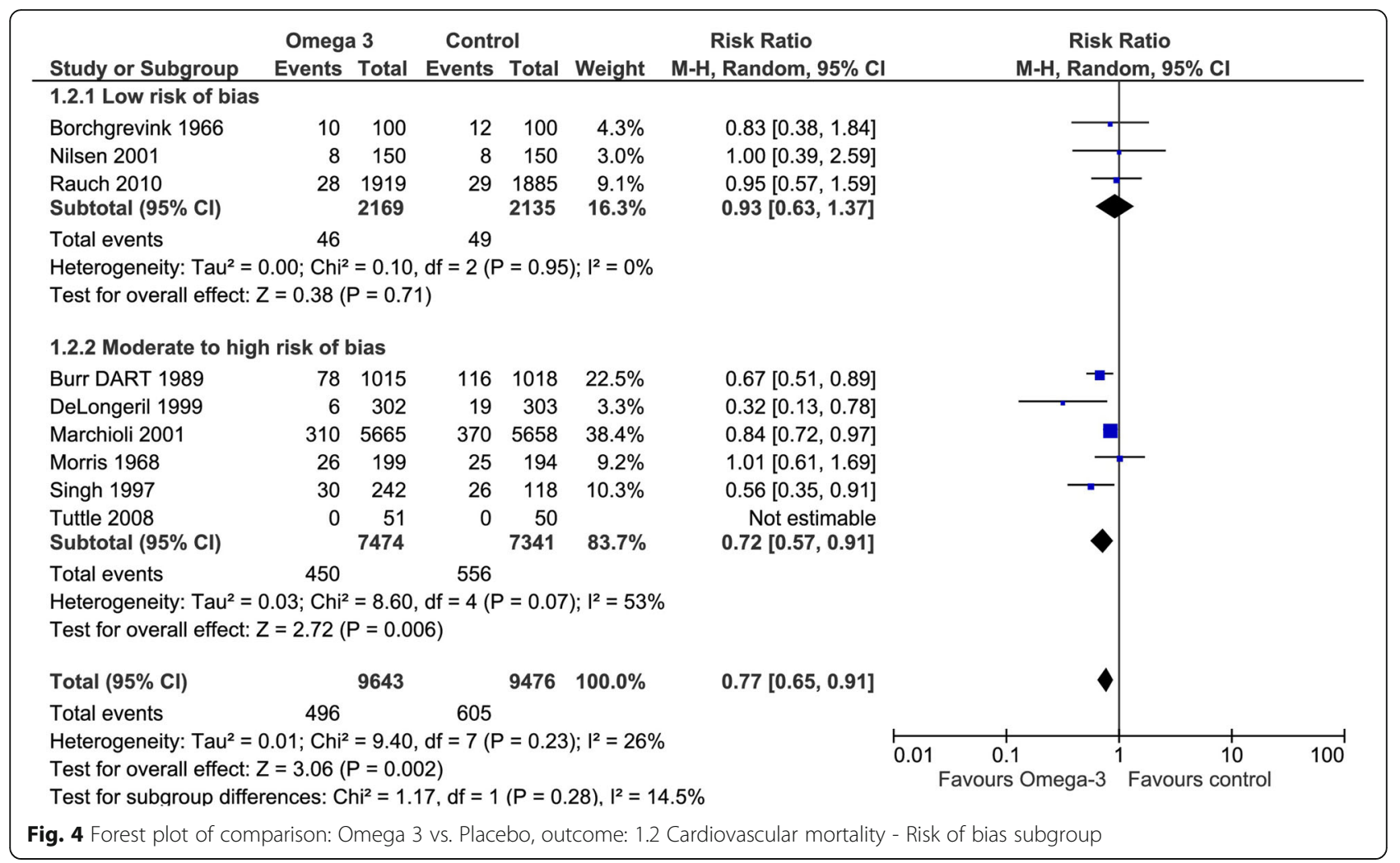

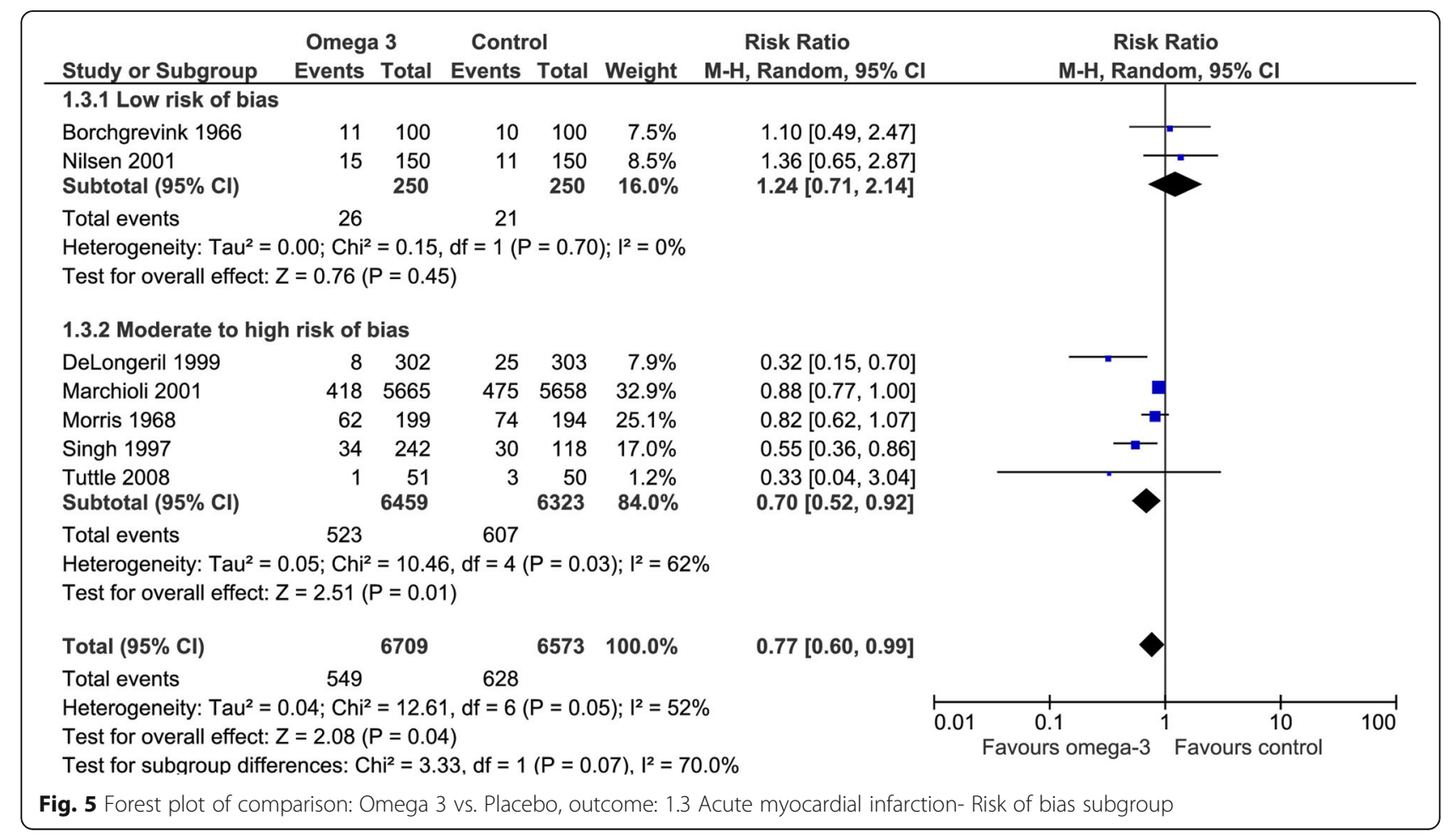




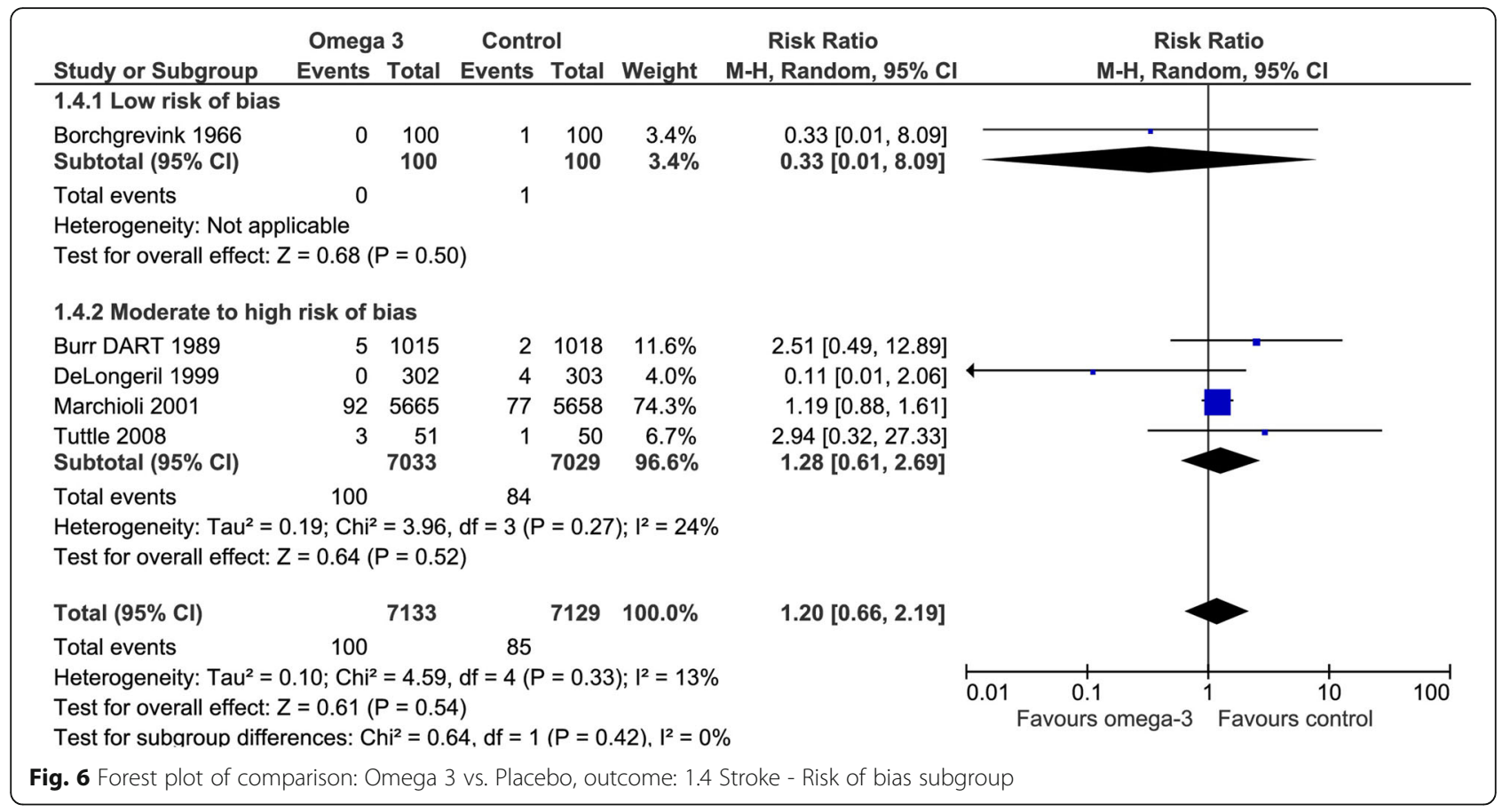

conclusions regarding this outcome. This information, consistent with the conclusions of other systematic reviews on the topic [38-40], can help decision makers by supporting the fact that the risk of significant negative effects related to omega 3 fatty acids cannot be ruled out.

Even though we observed a possible subgroup effect in favor of DHA-EPA fatty acids, this is mainly based in differences between studies, as opposed to differences in subgroups within studies, and the differential effect was mainly influenced by moderate/high risk of bias trials.
We consider that a true subgroup effect under these conditions is unlikely.

Our review has limitations,. Although we performed a thorough evidence search, we did not explored conference abstracts. Besides we did not include studies that randomized patients with AMI combined with other subpopulations (i.e patients with stroke) [41] as we could not gain access to individual patients' data or AMI subgroup results for any of those identified trials [42, 43].

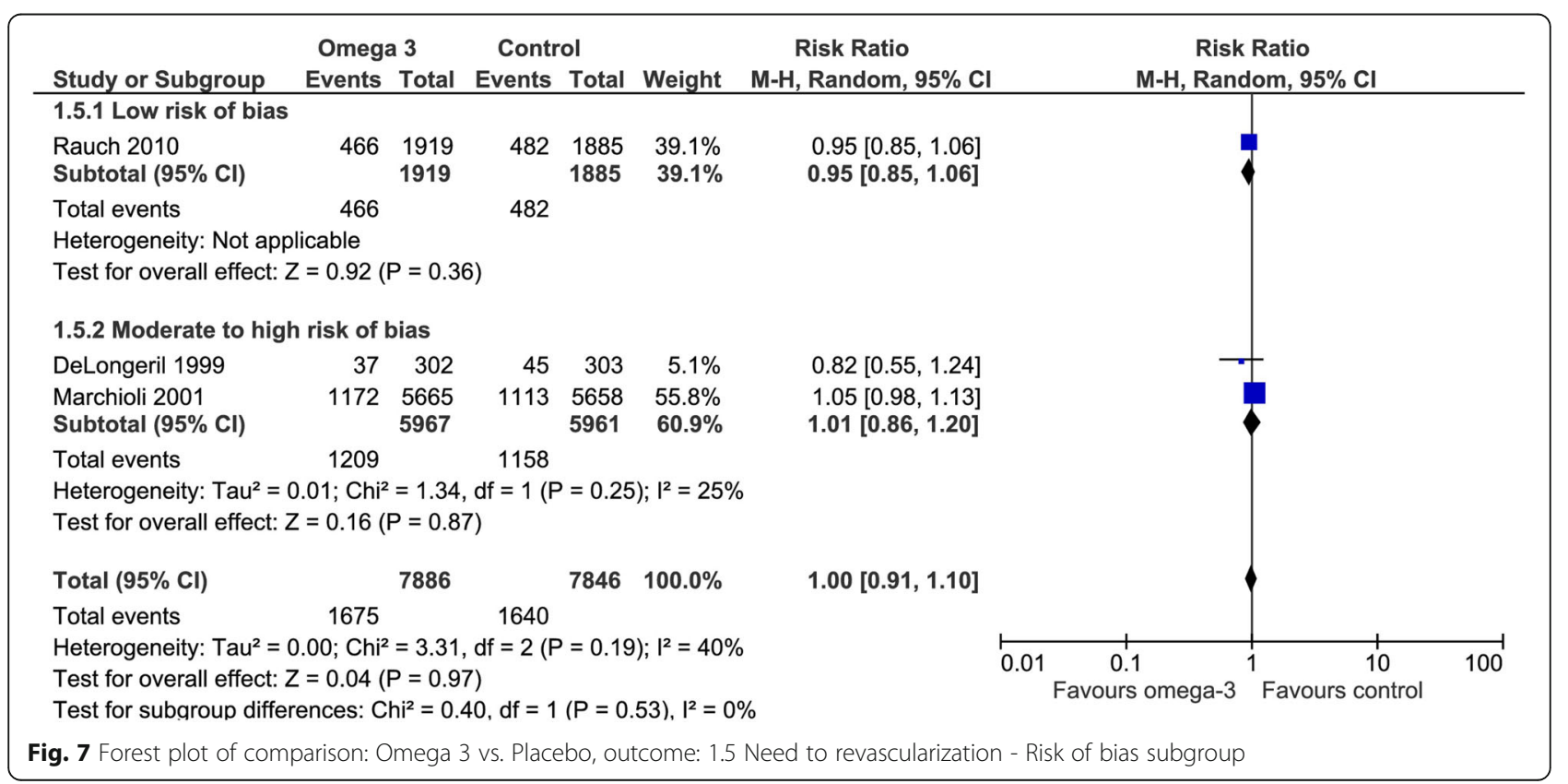




\begin{tabular}{|c|c|c|c|c|c|c|c|c|c|}
\hline Study or Subgroup & $\begin{array}{c}\text { Omega } \\
\text { Events }\end{array}$ & Omega 3 & Control & $\begin{array}{l}\text { ol } \\
\text { Total }\end{array}$ & Weight & $\begin{array}{c}\text { Risk Ratio } \\
\text { M-H, Random, } 95 \% \mathrm{Cl}\end{array}$ & \multicolumn{3}{|c|}{$\begin{array}{c}\text { Risk Ratio } \\
\text { M-H, Random, } 95 \% \mathrm{Cl} \\
\end{array}$} \\
\hline Kromhout 2010 & 173 & 3601 & 48 & 1236 & $45.1 \%$ & $1.24[0.90,1.69]$ & & & \\
\hline Rauch 2010 & 99 & 1919 & 84 & 1885 & $54.9 \%$ & $1.16[0.87,1.54]$ & & & \\
\hline Total $(95 \% \mathrm{Cl})$ & & 5520 & & 3121 & $100.0 \%$ & $1.19[0.97,1.47]$ & & 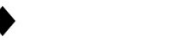 & \\
\hline Total events & 272 & & 132 & & & & & & \\
\hline $\begin{array}{l}\text { Heterogeneity: } \mathrm{Tau}^{2}= \\
\text { Test for overall effect: }\end{array}$ & $\begin{array}{l}.00 ; \mathrm{Chi}^{2} \\
=1.64(\mathrm{P}\end{array}$ & $\begin{array}{l}=0.09 \\
P=0.10\end{array}$ & $d f=1(P$ & $=0.76$ & $;\left.\right|^{2}=0 \%$ & & $\begin{array}{c}0.020 .1 \\
\text { Favours omega-3 }\end{array}$ & $\begin{array}{c}10 \\
\text { Favours control }\end{array}$ & 50 \\
\hline
\end{tabular}

Although we understand that we could have missed relevant information as a consequence of the mentioned limitations, we consider that improbable. Our systematic review has also particular strengths. First, it provides the most comprehensive and trustworthy body of evidence up to date, including studies that were not included in other recent prior $[19,20,22,23,28]$ reviews. While the conclusions of our systematic review in terms of the effects of the intervention are not different from the conclusions of some of the published reviews addressing similar questions, we believe that the analysis of the certainty of the evidence and the way in which we presented the results (following the GRADE approach) better reflects the trustworthiness of the information available, particularly regarding the absence of benefits in terms of mortality reduction and AMI recurrence.

As mentioned in the introduction, none of the published reviews on the topic, particularly the recently published Cochrane review [44], performed a complete and in-depth analysis of the effects of Omega 3 fatty acids on the population of patients with acute myocardial infarction. The most relevant differences of those SR with ours comprise: 1) None included all the available evidence [32, 44-46]; 2) Most did not perform a subgroup analysis considering the risk of bias of the included studies, which we believe, that in this particular scenario, is crucial to interpret the whole body of evidence $[33,46]$; 3) Most included patients with cardiovascular risk factors, stable angina pectoris or other cardiovascular conditions but without previous events; whereas we focused our question on the group of patients with acute myocardial infarction hypothesizing that the Ingestion of omega-3 PUFAs including EPA and DHA may result in more significant benefits by attenuating the inflammatory response triggered by the myocardial injury $[33,44,45]$.

The results and conclusions of those published reviews are inconsistent. While some report a positive effect of omega 3 fatty acids and even recommend its use [47], others claim that there is not enough evidence about the benefits of the intervention [44, 45]. One of the most recent reviews, published by Aung et al. [48], deserves a detailed description. Although the authors appropriately analyzed the results considering the risk of bias of the primary studies, they failed to include most of the evidence related to patients with previous MI (9 of 11 studies) [19-27], see Additional file 1: Appendix 1). The authors concluded that omega- 3 fatty acids had no significant association with fatal or nonfatal coronary heart disease or any major vascular events. Similar considerations can be made regarding the Cochrane review [44]. Our results strengthen the ones seen in the reviews of Cochrane and Aung by expanding their findings to the high-risk subgroup of patients with previous MI.

\section{Conclusion}

Omega 3 fatty acid supplementation probably yields no benefit to patient important outcomes for individuals with previous AMI. The results of our systematic review would provide useful information to panels aiming to elaborate recommendations for the management of patients with previous AMI.

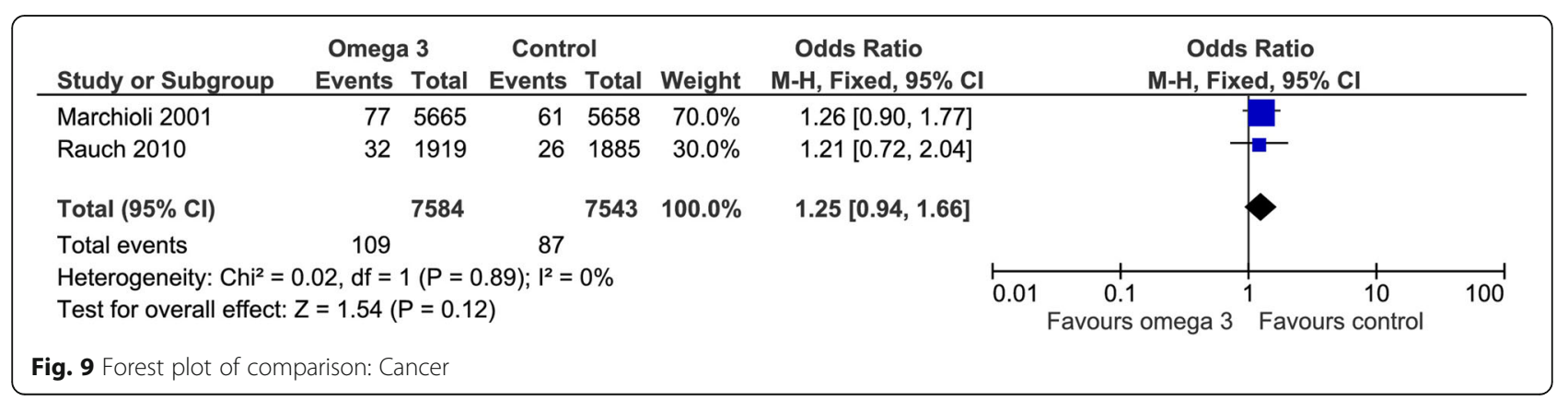




\section{Strengths and limitations of this study}

- The present systematic review provides estimations regarding the efficacy of supplemental omega 3 fatty acids in the context of myocardial infarction secondary prevention paying special attention to the risk of bias of the included studies

- Unlike most of the published reviews we focused in the population of patients that had suffered an acute myocardial infarction

- It provides the most comprehensive and trustworthy body of evidence up to date, including studies not included in any other published reviews $[19,20,22$, $23,28]$.

- We performed a thorough analysis of the information and identified significant differences in the results of the primary studies that could be explained by methodological limitations in some of them.

\section{Additional file}

Additional file 1: Appendix 1. Recently published relevant systematic reviews evaluating the intervention. Appendix 2 . Details of the full search strategies. Appendix 3. Funnel plot of the All-cause mortality comparison Appendix 4. Missing Outcome Data - Sensitivity analyses. (DOCX 106 kb)

\section{Abbreviations}

ALA: Alpha-linolenic acids; AMI: Acute myocardial infarction; Cl: Confidence interval; DHA: Docosahexaenoic acid; DPA: Docosapentaenoic acid; EPA: Eicosapentaenoic acid; GRADE: Grading of recommendations assessment, development and evaluation; PUFA: Poly-Insaturated fatty acids; $\mathrm{RCT}$ : Randomized controlled trials; RD: Risk difference; RR: Relative risk; RRD: Relative risk difference; SR: Systematic review

\section{Acknowledgments}

The authors thank Juan Martín Criniti and Juan Ignacio Ruiz for their help in the quality assessment of the primary studies and the Southern American Center of Excellence of Cardiovascular Health (CESCAS).

\section{Funding}

The group recieved no external funding. Publication fees were funded by Hugo Norberto Catalano, Ariel Izcovich, Federico Popoff and Giselle Balaciano.

\section{Availability of data and materials}

Not applicable.

\section{Authors' contributions}

PF, BG, BA, CD, IV, CHN, IA. FP: study selection and data extraction, quality assessment of the primary studies, statistical analysis, writing of manuscript. GB: study selection and data extraction, quality assessment of the primary studies, statistical analysis, writing of manuscript. AB: correction of manuscript. DC: Literature review. Vl: correction of manuscript. HNC: correction of manuscript. Al: quality assessment of the primary studies, discrepancies resolution, statistical analysis, correction of manuscript. All authors have read and approved the manuscript.

\section{Ethics approval and consent to participate}

Not applicable.

\section{Consent for publication}

Not Applicable.

\section{Competing interests}

The authors declare that they have no competing interests.

\section{Publisher's Note}

Springer Nature remains neutral with regard to jurisdictional claims in published maps and institutional affiliations.

\section{Author details}

${ }^{1}$ Ministry of health, Buenos Aires, Argentina. ${ }^{2}$ National Ministry of Health of Argentina, Hospital Alemán, Buenos Aires, Argentina. ${ }^{3}$ Institute for Clinical Effectiveness and Health Policy, Buenos Aires, Argentina.

Received: 27 July 2018 Accepted: 22 April 2019

Published online: 04 June 2019

\section{References}

1. Calabresi L, Villa B, Canavesi M, Sirtori CR, James RW, Bernini F, et al. An omega-3 polyunsaturated fatty acid concentrate increases plasma highdensity lipoprotein 2 cholesterol and paraoxonase levels in patients with familial combined hyperlipidemia. Metabolism. 2004;53(2):153-8.

2. Foundation., B.N. n-3 fatty acids and health: briefing paper. London: British Nutrition Foundation; 1999.

3. Bhatnagar D, Durrington PN. Omega-3 fatty acids: their role in the prevention and treatment of atherosclerosis related risk factors and complications. Int J Clin Pract. 2003;57(4):305-14.

4. Thies F, Garry JM, Yaqoob P, Rerkasem K, Williams J, Shearman CP, et al. Association of n-3 polyunsaturated fatty acids with stability of atherosclerotic plaques: a randomised controlled trial. Lancet. 2003;361:477-85.

5. Cawood AL, Ding R, Napper FL, Young RH, Williams JA, Ward MJ, Gudmundsen O, Vige R, Payne SP, Ye S, Shearman CP, Gallagher PJ, Grimble RF, Calder PC. Eicosapentaenoic acid (EPA) from highly concentrated n-3 fatty acid ethyl esters is incorporated into advanced atherosclerotic plaques and higher plaque EPA is associated with decreased plaque inflammation and increased stability. Atherosclerosis. 2010;212(1):252-9.

6. Bang HO, Dyerberg J. Plasma lipids and lipoproteins in Greenlandic west coast eskimos. ActaMedicaScandinavica. 1972;192:85-94.

7. Bang HO, Dyerberg J, Hjorne N. The composition of food consumed by Greenland Eskimos. ActamedicaScandinavica. 1976;200:69-73.

8. CADTH - Grey Matters: a practical tool for searching health-related grey literature. https://www.cadth.ca/resources/finding-evidence/grey-matters. Accessed 5 May 2019

9. National Center for Biotechnology Information. PubChem Compound Database; CID=446284. https://pubchem.ncbi.nlm.nih.gov/compound/ 446284. Accessed 5 May 2019.

10. University, C.G.a.M., Tools to assess risk of bias in cohort and case control studies; randomized controlled trials; and longitudinal symptom research studies aimed at the general population. 2013.

11. Guyatt GH, Oxman AD, Kunz R, Vist GE, Falck-Ytter $Y$, Schünemann HJ. GRADE Working Group. What is "quality of evidence" and why is it important to clinicians? BMJ. 2008;336:995-8.

12. Guyatt $\mathrm{GH}$, Oxman $A D$, Kunz R, et al. GRADE guidelines 6 . Rating the quality of evidence-imprecision. J Clin Epidemiol. 2011;64:1283-93.

13. Schünemann H, Brożek J., Guyatt G, Oxman A, GRADE handbook for grading quality of evidence and strength of recommendations. 2013.

14. Higgins JPT, Green S, Cochrane Handbook for Systematic Reviews of Interventions Version 5.1.0 [updated March 2011]. The Cochrane Collaboration; 2011. Available from https://training.cochrane.org/handbook.

15. National Center for Biotechnology Information. PubChem Compound Database; CID=5280934. https://pubchem.ncbi.nlm.nih.gov/compound/ 5280934. Accessed 5 May 2019

16. Akl EA, et al. Addressing dichotomous data for participants excluded from trial analysis: a guide for systematic reviewers. PLoS One. 2013;8(2):e57132.

17. Guyatt GH, Ebrahim S, Allonso-Coello P, Johnston BC, Mathioudakis AG, Briel M, Mustafa RA, Sun X, Walter SD, Heels-Ansdell D, Neumann I, Kahale LA, Iorio A, Meerpohl J, Schünemann H, AkI EA. GRADE guidelines 17: assessing the risk of bias associated with missing participant outcome data in a body of evidence. J Clin Epidemiol. 2017;87:14-22

18. Kromhout $\mathrm{D}$, et al n-3 fatty acids and cardiovascular events after myocardia infarction. N Engl J Med. 2010;363(21):2015-26. 
19. de Lorgeril M, et al. Mediterranean diet, traditional risk factors, and the rate of cardiovascular complications after myocardial infarction: final report of the Lyon diet heart study. Circulation. 1999;99(6):779-85.

20. Tuttle KR, et al. Comparison of low-fat versus Mediterranean-style dietary intervention after first myocardial infarction (from the heart Institute of Spokane Diet Intervention and Evaluation Trial)+†conflicts of interest: Dr. Bibus has received consulting fees from companies that make fish oil, Coromega Co., vista, CA, and Enreco, Inc., Newton, WI. He also has equity ownership in lipid technologies, LLC, Austin, MN, the company that performed the plasma fatty acid analysis. Dr. Bibus owns patents related to lipid therapy. He has served as an expert witness regarding lipids and fatty acids. Am J Cardiol. 2008;101(11):1523-30.

21. Burr ML, et al. Effects of changes in fat, fish, and fibre intakes on death and myocardial reinfarction: diet and reinfarction trial (DART). Lancet. 1989; 2(8666):757-61.

22. Borchgrevink CF, et al. Absence of prophylactic effect of linolenic acid in patients with coronary heart-disease. Lancet. 1966;2(7456):187-9.

23. Morris JN. Controlled trial of soya-bean oil in myocardial infarction, REPORT OF A RESEARCH COMMITTEE TO THE MEDICAL RESEARCH COUNCIL. Lancet. 1968;2(7570):693-9.

24. Marchioli R, et al. Efficacy of n-3 polyunsaturated fatty acids after myocardial infarction: results of GISSI-Prevenzione trial. Gruppo Italiano per lo Studio della Sopravvivenza nell'Infarto Miocardico. Lipids. 2001;36(Suppl):S119-26.

25. Nilsen DW, et al. Effects of a high-dose concentrate of $n-3$ fatty acids or corn oil introduced early after an acute myocardial infarction on serum triacylglycerol and HDL cholesterol. Am J Clin Nutr. 2001;74(1):50-6.

26. Rauch $\mathrm{B}$, et al. OMEGA, a randomized, placebo-controlled trial to test the effect of highly purified Omega-3 fatty acids on top of modern guideline-adjusted therapy after myocardial infarction. Circulation. 2010;122(21):2152-9.

27. Singh RB, et al. Randomized, double-blind, placebo-controlled trial of fish oil and mustard oil in patients with suspected acute myocardial infarction: the Indian experiment of infarct survival--4. Cardiovasc Drugs Ther. 1997;11(3): 485-91

28. Heydari B, Abdulla S, Pottala JV, Shah R, Abbasi S, Mandry D, Francis SA, Lumish H, Ghoshhajra BB, Hoffman U, Appelbaum E, Feng JH, Blankstein R, Steigner M, JP MC, Harris W, Antman EM, Jerosch-Herold M, Kwong RY. Effect of Omega-3 Acid Ethyl Esters on Left Ventricular Remodeling After Acute Myocardial Infarction: The OMEGA-REMODEL Randomized Clinical Trial. Circulation. 2016;134(5):378-91.

29. Grundt H, Nilsen DW, Hetland $\varnothing$, Mansoor MA. Clinical outcome and atherothrombogenic risk profile after prolonged washout following longterm treatment with high doses of n-3 PUFAs in patients with an acute myocardial infarction. Clin Nutr. 2004 Aug;23(4):491-500.

30. Smith J, Godlee F. Investigating allegations of scientific misconduct Journals can do only so much; institutions need to be willing to investigate. BMJ. 2005;6:331-245.

31. C W. Suspected research fraud: difficulties of getting at the truth. BMJ. 2005; 30(33):281-8.

32. Mercury Levels in Commercial Fish and Shellfish (1990-2012). https://www. fda.gov/food/metals/mercury-levels-commercial-fish-and-shellfish-1990-2012. Accessed 5 May 2019.

33. Hooper L, et al. Omega 3 fatty acids for prevention and treatment of cardiovascular disease, in Cochrane Database of Systematic Reviews, C. The Cochrane. Chichester: John Wiley \& Sons, Ltd; 2004.

34. Liem AKD, T.R. Dioxins: chemical analysis, exposure and risk assessment. 1st ed. Utrecht: Universiteit; 1997. (ISBN 903932012 8)

35. UK., F.S.A., Dioxins and PCBs in the UK diet: 1997 Total diet study samples. Food Surveillance Information Sheet. 2000(Vol. 4/00. [ FSIS 4/00]).

36. Committee on toxicity of chemicals in food, consumerproducts and the environmentupdated - cot statement on a survey of mercury in fish and shellfish. https://cot.food.gov.uk/sites/default/files/cot/cotstmercuryfish.pdf. Accessed 5 May 2019.

37. Fernandes AR, Rose M, White S, Mortimer DN, Gem M. Dioxins and polychlorinated biphenyls (PCBs) in fish oil dietary supplements: Occurrence and human exposure in the UK. Food Addit. Contam. 2006;23:939-47.

38. Wen YT, Dai JH, Gao Q. Effects of Omega-3 fatty acid on major cardiovascular events and mortality in patients with coronary heart disease: A meta-analysis of randomized controlled trials. Nutr Metab Cardiovasc Dis. 2014;24:470-475.

39. Aucoin M, Cooley K, Knee, Fritz Heidi, Balneaves GL, Breau R, Fergusson D, Skidmore B, Wong R, Seely D. Fish-Derived Omega-3 Fatty Acids and
Prostate Cancer: A Systematic Review, Integr Cancer Ther. 2017;16(1):32-62. https://doi.org/10.1177/1534735416656052.

40. MacLean CH, Newbery SJ, Mujica WA, Issa A, Khanna P, Lim YW, et al. Effects of omega-3 fatty acids on cancer. Evidence Report/Technology Assessment. Rockville (MD): Agency for Healthcare Research and Quality; 2005. p. E0102. Report No: 113(AHRQ Publication No: 05).

41. Galan P, et al. Effects of B vitamins and omega 3 fatty acids on cardiovascular diseases: a randomised placebo controlled trial. BMJ. 2010; $341: c 6273$.

42. Tavazzi L, Maggioni AP, Marchioli R, Barlera S, Franzosi MG, Latini R, Lucci D, Nicolosi GL, Porcu M, Tognoni G, Gissi-HF Investigators. Effect of n-3 polyunsaturated fatty acids in patients with chronic heart failure (the GISSIHF trial): a randomised, double-blind, placebo-controlled trial. Lancet. 2008; 372(9645):1223-30.

43. Yokoyama M, Origasa $H$, Matsuzaki M, Matsuzawa $Y$, Saito $Y$, Ishikawa $Y$, Oikawa S, Sasaki J, Hishida H, Itakura H, Kita T, Kitabatake A, Nakaya N, Sakata T, Shimada K, Shirato K, Japan EPA lipid intervention study (JELIS) Investigators. Effects of eicosapentaenoic acid on major coronary events in hypercholesterolaemic patients (JELIS): a randomised open-label, blinded endpoint analysis. Lancet. 2007;369(9567):1090-8.

44. Abdelhamid AS, Brown TJ, Brainard JS, Biswas P, Thorpe GC, Moore HJ, Deane KH, AlAbdulghafoor FK, Summerbell CD, Worthington HV, Song F, Hooper L. Omega-3 fatty acids for the primary and secondary prevention of cardiovascular disease. Cochrane Database Syst Rev. 2018;7:CD003177.

45. Kwak SM, MS, Lee YJ, Seo HG. Efficacy of omega-3 fatty acid supplements (Eicosapentaenoic acid and docosahexaenoic acid) in the secondary prevention of cardiovascular disease a meta-analysis of randomized, doubleblind, placebo-controlled trials. Arch Intern Med. 2012;172(9):686-94.

46. National Clinical Guideline, C. Myocardial infarction: cardiac rehabilitation and prevention of further MI NICE guidelines [CG172]. 2013.

47. Smith SC Jr, Allen J, Blair SN, Bonow RO, Brass LM, Fonarow GC, Grundy SM, Hiratzka L, Jones D, Krumholz HM, Mosca L, Pasternak RC, Pearson T, Pfeffer MA, Taubert KA; AHA/ACC; National Heart, Lung, and Blood Institute. AHA/ ACC guidelines for secondary prevention for patients with coronary and other atherosclerotic vascular disease: 2006 update: endorsed by the National Heart, Lung, and Blood Institute. Circulation. 2006 May 16;113(19): 2363-72.

48. Aung T, Halsey J,Kromhout D. Associations of Omega-3 Fatty Acid Supplement Use With Cardiovascular Disease RisksMeta-analysis of 10 Trials Involving 77917 Individuals. JAMA Cardiol. 2018;3(3):225-233. https://doi. org/10.1001/jamacardio.2017.5205.

\section{Ready to submit your research? Choose BMC and benefit from:}

- fast, convenient online submission

- thorough peer review by experienced researchers in your field

- rapid publication on acceptance

- support for research data, including large and complex data types

- gold Open Access which fosters wider collaboration and increased citations

- maximum visibility for your research: over $100 \mathrm{M}$ website views per year

At $\mathrm{BMC}$, research is always in progress.

Learn more biomedcentral.com/submissions 